\title{
Designing Internet of Things System for Checking Cattle Rustling in Nigeria
}

\author{
Eke B. O. \\ Dept. of Computer Science, \\ University of Port Harcourt, \\ Port Harcourt, Nigeria
}

\author{
Egbono $\mathrm{F}$. \\ Dept. of Computer Science, \\ University of Port Harcourt, \\ Port Harcourt, Nigeria
}

\begin{abstract}
In recent time the stealing of grazing cattle have increased in Nigeria giving the herdsmen the excuse of carrying dangerous weapons. Many soldiers are deployed to watch cattle instead of engaging them in more defense resourceful ventures. Internet of Things (IoT) is a computer and internet technology that creates an environment in which objects, animals and people are provided with unique identifiers and the ability to transfer data over a network without requiring human-to-human or human-to-computer interaction. This paper proposes a design of an IoT system that will allow the implantation of sensors in the cows so that their movement can be easily monitored. The system will ensure that when rustling occurs the application can be used in tracking the criminals.
\end{abstract}

\section{General Terms}

Cattle Rustling, Herdsmen, Internet, Tracking

\section{Keywords}

Internet of Things, Sensors, Mobile Devices

\section{INTRODUCTION}

In modern time, various gadgets and physical components are interconnected via the internet as well as other local or wide area network. . In addition we currently see a big rise in the interconnection of physical entities referred to as "Things" in the new technology - Internet of Things (IoT) in which real world objects have an individual digital presence [1]. In IoT technology, physical objects are uniquely identified and defined in a standardized way which facilitates access to and interaction with them [2]. IoT has evolved from the convergence of wireless technologies, micro-electromechanical systems (MEMS) and the Internet. The concept may also be referred to as the Internet of Everything including living and non-living things. In the Internet of Things (IoT) paradigm, many of the objects that surround us will be on the network in one form or another. The Radio Frequency Identification (RFID) Development community had been in the forefront of developing and improving the technology and it has recently become more relevant to the practical world largely because of the growth of mobile devices, embedded and ubiquitous communication, cloud computing and data analytics. In IoT world billions of objects can sense, communicate and share information, all interconnected over public or private Internet Protocol (IP) networks. These interconnected objects have data regularly collected, analyzed and used to initiate action, providing a wealth of intelligence for planning, management and decision making. This is the world and technology of Internet of Things (IOT).

Since then, many visionaries have seized on the phrase "Internet of Things" to refer to the general idea of things, especially everyday objects, that are readable, recognizable, locatable, addressable, and/or controllable via the Internet, irrespective of the communication means (whether via RFID, wireless LAN, wide- area networks, or other means).

In recent time, things that are not just electronic devices or the products of higher technological development but things that we do not ordinarily think of as electronic at all, such as animals and humans are connectable. If they are connectable then the benefit of location identification and other connectivity benefits can be derivable from the interconnectivity.

In this paper we propose a design of an Internet of Things application layer that will link with the implanted sensors in their cows and then be easily used by herdsmen on their mobile devices to keep track of their herds. The system will enable them to know when cattle rustlers are attacking and to location they may have taken the cows to, who may have bought the cows and everything about the cows. Our design will provide the layer for the combination of physical mobile interactions with the Internet of Things infrastructure and technological devices.

\section{THE SYSTEM INFRASTUCTURE \\ 2.1 Mobile System}

A mobile device is a handheld or portable device that is made to be both compact and lightweight. New data storage, processing and display technologies have allowed these smal devices to do nearly anything that had previously been traditionally done with larger personal computers. These devices use broadband system in carrying out complex activities. A mobile broadband system (MBS) is the infrastructure that provides Internet access to mobile and handheld device users. It is a system used by mobile service providers to deliver high-speed broadband Internet access to remotely connected users [3].

These networks provide for the clients easy interaction, access to resources, business interactions and information dissemination. It also provides means of transmitting various needs to all the points where solutions are needed and can be provided [4].

Many mobile device users carry out more complex and ubiquitous information processing and management activities and mobile applications offer increasingly sophisticated methods to capture information, to make use of context information and to interact directly with objects in the real world. On the other hand physical objects are increasingly associated with digital information through the augmentation with visual [5] and wireless markers such as RFID tags.

In this context physical mobile interactions allow users toselect virtual information and invoke services through the interaction with objects in the real world [6]. Currently there are several approaches for the provision of applications that take such interactions into account. Most of them are proprietary, 
designed for a special application area or interaction technique and provide no generic concept for the description of real world services..

\subsection{Sensor}

Some communication system relies heavily on the links interaction between two interacting devices on an internetwork. In most times sensors are used as medium and sometimes transmitters are also used when radio communication is integrated. The system signal input is usually reflected or outputted by these mediums for the system application to interpret.

Sensor is a device which provides a usable output in response to a specified measurand. A sensor acquires a physical quantity and converts it into a signal suitable for processing. The signal can be digital or electrical depending on the network. Sensors contain active element called a transducer, figure 1 illustrates small sensors that can be embedded into animals with different sizes even though they have the same capacities.

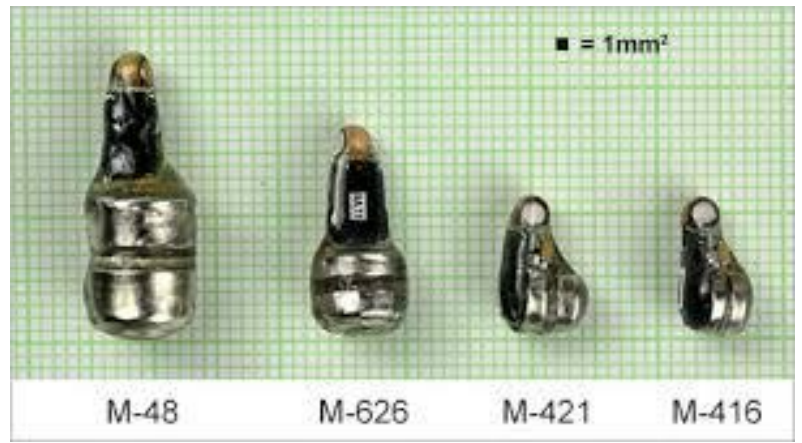

Fig. 1: Sensors of same capacity but different sizes.

Sensors can acquire the following physical quantities which include Biological, Chemical, Electric, Electromagnetic and Heat/Temperature. Other physical quantities acquirable by sensors include: Magnetic, Mechanical motion (displacement, velocity, acceleration), Optical and Radioactivity.

In this work we are particularly interested in extracting Biological and Mechanical quantities from the sensors in our internetworking space. The stimuli measured in mechanical quantities are Position, Velocity, Acceleration, Force, Strain, Stress, Pressure, and Torque. These quantities vary and their values are actually what the system may need as its input [7]. They are actually needed to provide automation.

In the design of the system a cost effective but efficient sensor is recommended so that implementation cost will not inhibit the operation of the system.

\subsection{Tracking Circuits (Transmitters)}

The tracking circuit of the sensor need to be a circuit that is very compact and consumes almost no power. It should be small enough to be hidden in anything you suspect will be lost or stolen. By using a mercury switch or grasshopper a cattle can be "primed" for the time when it is moved and it can be tracked with the application layer. A grasshopper is a switch that is ready to go off at any time. A piece of plastic is placed between two switch contacts to keep them apart and connected to a thread. When the object is moved, the system is turned ON. System trigger like this would make an ideal detector to track down anything going astray. By attaching it to the cows under surveillance, you can follow its removal (rustle) and maybe turn up quite a few surprises. The Tracker transmits a very short burst of carrier which produces a blank spot on the dial - commonly called "silence." Normally, a lot of background noise called "snow" is picked up by a system network when it is tuned to a frequency between the stations. The change between silence and snow produces a "click" or "mow" and this is the noise produced by the cow emitted about twice a second [8].

In figure 2, the tracking circuit consists of two building blocks - both are oscillators. The first operates at a very low rate (low frequency - about $2 \mathrm{~Hz}$ ) and the other operates at approx $90 \mathrm{MHz}$. The first is a square-wave oscillator with a very short "ON time," while the other is a sine-wave. The only thing they have in common is a "feedback component," to create and maintain oscillation. In all other respects they are different [8].

The first block is a 2-transistor Pulse generator and the second is an $\mathbf{R F}$ oscillator. The whole circuit takes very little average current as it is active for very short bursts. But the current is high during the short bursts of operation and we need to check the current taken during the bursts, to make sure it is as low as possible.

To prevent high current, the Pulse Generator circuit is separated from the RF Oscillator with a $1 \mathrm{k}$ resistor. To give the Pulse Generator its own separate "Power Supply" a $22 \mathrm{u}$ electrolytic was added. The diagram below shows the separate "Power Supply" for the Pulse Generator. The $22 \mathrm{u}$ charges via the $1 \mathrm{k}$ resistor and when the Pulse Generator circuit requires its "high current," the $22 \mathrm{u}$ delivers the energy. This feature is called Stage Separation or Block Isolation and separates the power requirements of a "building block" from the rest of the circuit. The $1 \mathrm{k}$ and $22 \mathrm{u}$ electrolytic have been connected exactly like a "delay circuit" but they are not used as a delay feature in this arrangement. The $1 \mathrm{k}$ slowly charges (in relative terms) the $22 \mathrm{u}$ and the Pulse Circuit draws a high amount of energy from the $22 \mathrm{u}$ for a very short period of time. The voltage across the $22 \mathrm{u}$ drops slightly and when the Pulse circuit switches "off," the $22 \mathrm{u}$ charges to maximum voltage.

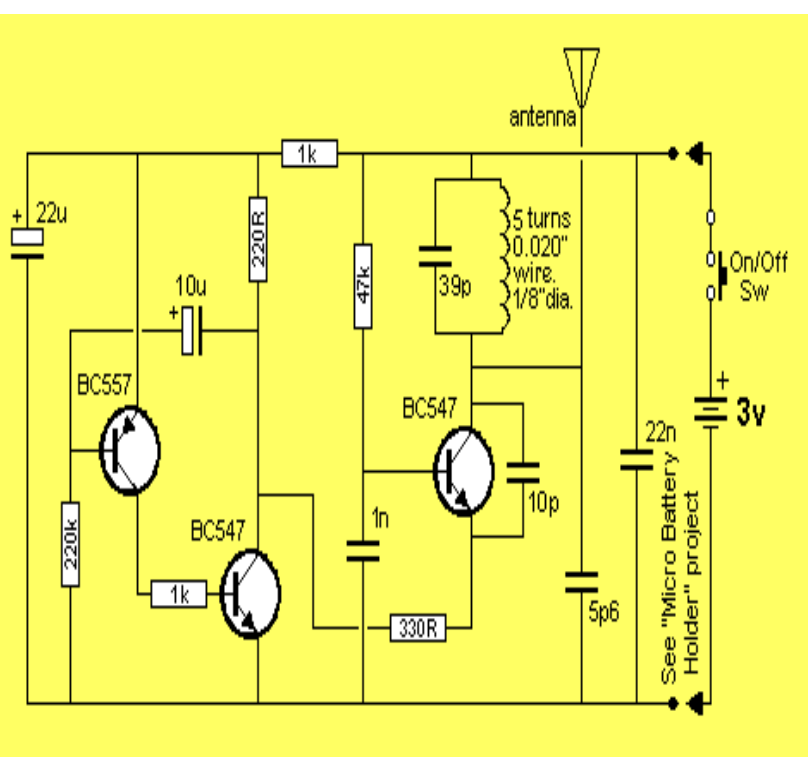

Fig. 2: A transmitter circuit for tracking object.(Adopted: [8])

The relative advantage explained about the sensor with the transmitter circuit make it unavoidable for the adaption of this sensor for the purpose of the research. These will make the sensor cheap to use per cow and effective in power to allow it last long before any need for possible replacement. 


\subsection{Internetwork Devices}

Internetworking is the practice of connecting a device network with other device networks through the use of gateways that provide a common method of routing information packets between the networks. The resulting system of interconnected networks are called an internetwork, or simply an internet. The Internet is the largest example of an internetworking and it is based on many underlying hardware technologies, but unified by an internetworking protocol standard, the Internet Protocol Suite, often also referred to as TCP/IP [9].

Internetworking device or connectivity device are devices used to make physical network connections. They do not make changes to the data or transmission route. Connectivity devices operate at the physical layer of the Open Systems Interconnection (OSI) model. Internetworking devices move data across a network. They may direct data to specific locations within the network and/or convert data into alternative formats. Internetworking devices operate at OSI layers above the physical layer. Understanding the functions of these devices and how they fit within the OSI model will help you learn how networks function.

The internetworking devices needed by the system largely depend on how the circuit if wired. If the electrical characteristics of various networks for the sensor and the mobile device are different, media filter adapter connectors may be attached to the mobile device to make the connections possible. But if the sensor circuit includes an internal network interface card (a printed circuit board) then TCP/IP protocol can simply link the devices. The interface card provide the physical connection and circuitry required to access the mobile network. The shortest way recommended in this work is the use of internal transceivers (the combination transmitter and receiver) in the circuit. It assists in a quick transmission and reception of signals and connects a mobile device to the network.

\section{CATTLE RUSTLING IN NIGERIA}

We have reviewed the key technology and devices needed to design our system but we need to look at the area of deployment and examine how activities of the hoodlums (cattle rustlers) are carried out. We believe that the knowledge will assist not only in the sensor selection but in the usability concern of our application layer design. If this aspect is neglected we may end up with a good system that the cattle herdsmen will not be able to use.

Cattle rustling refers to the stealing of grazing cattle [10]. The term is of the historical United States colloquial etymology in which context pioneer farmers lost cattle while grazing on huge ranges that were difficult to patrol for policing (Microsoft Students Encarta, 2008 DVD). Traditionally, cattle rustling has been driven by the criminal intent to expropriate cow for meat or for sale [10]. Pertinently, it must have served as a means of primitive accumulation of cow-herd in the contexts of subsistence and commercial pastoralism [11].

Over the years, cattle rustling has evolved into a pattern of organized crime with immense criminal sophistication and efficiency. Hence, contemporary cattle rustlers operate with modern weaponry and their operations are marked by translocational and trans-national syndication [12]. This clearly underscores cattle rustling as a typical and prominent instance of 'underworld franchise' in contemporary societies [13].

Cattle's rustling is a global phenomenon. It has manifested in various scales and dimensions across the nations. For instance, in Scotland, 300 cattle were reported to have been rustled by the end of 2013, leading to the declaration of cattle rustling as a national emergency by the government [10].

In Nigeria, the prevalence of cattle rustling has been widespread, particularly in the northern part of the country where cattle raring obtains as a dominant agricultural practice. Recent developments tend to have implicated cattle rustling in the rising wave of violence in northern Nigeria, as exemplified in the phenomenon of 'Boko Haram' an insurgency in Nigeria and herder/farmer conflicts[14]. This scenario has accentuated the significance of cattle rustling as a fundamental national security problem in Nigeria. It is against this backdrop that this paper examines the phenomenon of cattle rustling in northern Nigeria and proposes an IoT system design that can assist the herds men in tracking their cows and assisting security men in arresting the situation.

\subsection{Brief Review of Causes of Cattle Rustling}

The challenges of cattle rustling is not peculiar to Nigeria alone in countries like Niger, Chad and even Kenya they are also common. In the North region of Kenya there are such conflicts which are thought to be part and parcel of a pastoral culture and livelihood of resident communities. More so conflicts between Marakwet and Pokot communities are deemed to be resource based resulting from competition over pasture and water [15]. Cattle rustling are carried out by forceful raiding of livestock from one community by another using guns and leaving behind destruction of property and loss of lives. This concept should be understood alongside cattle raids which involve stealing livestock from one community by another without destroying property or killing people. Prior to 1990 cattle raids are meant to steal livestock, by scaring away their owners, but cattle rustling involves destruction of property and murder [15]. Cattle rustling have become a commercial entity along the boundaries of pastoral communities and stolen livestock are never recovered. The actors in cattle rustling involve politically linked and power wielding personalities sometimes not pastoralist [15], thus before we present our technological design, we will review the nature and causes of cattle rustling among cattle farmers.

Proliferation of illegal weapons among cattle herdsmen had increased the severity of cattle rustling. The causes of conflicts are blamed on invasion of pasture/grazing lands by a raiding groups. Once the raids intensified, other communities who lay claim the grazing field were forced to purchase firearms to counter attack their raiders. This leads to community and tribal arms race, attack, reprisal attack and counter attack.

\subsection{Perspectives on Cattle Rustling}

Conceptually, the term 'cattle rustling' appears to have evolved into a more or less standardized, specialist nomenclature. Hence, the concept is almost universally applied to designate the act of stealing cattle from a grazing herd notwithstanding the motivation or contextual specifics. Some recent studies have, however, used the notion of 'cattle raid' to denote the same reality [16]. Cattle rustlers are principally motivated by the criminal intent to expropriate grazing cattle for meat or for sale [17]. As a corollary, cattle rustling have also been largely motivated by the quest for primitive accumulation of capital and untaxed wealth [18]. In this sense, cattle rustling passes for a typical instance of organized crime [14]. An organized crime is a criminal enterprise involving discernible hierarchical social networking and syndication [18]

It is clear that cattle rustling as an organized crime, among other things, shows the level of sophistication and criminal 
efficiency that characterizes its contemporary manifestations. It is therefore clear that cattle rustling have evolved into a national crises which must be checked before it totally destroys the livelihood of the people. In its apparent global dynamics of sophistication and degeneration, cattle rustling has been associated with spiral violence in different parts of the world today [17].

Cattle rustling in Nigeria is a major problem in rural areas across different states and the groups involved in the crime are often armed and pose a threat to the state as their activities may lead to violent conflicts between communities or often support the Islamic insurgence known as 'Boko Haram' groups that have been causing human menses in the North-east of the countries.

With reference to the contemporary trends and dynamics of cattle rustling in Kenya, Greiner opines: "[Cattle] raiding among northern Kenya's pastoralists has changed profoundly in the last decades. Fought with modern weaponry and often extreme violence, raiding is increasingly enmeshed in politicized claims over administrative boundaries, struggles for exclusive access to land and attempts to establish or safeguard ethnically homogenous election base" [16].

The association of cattle rustling with political struggles has been evident in some parts of Africa. For instance, in 2013, many people were killed or wounded in series of massive cattle raids perpetrated by rebels in Jonglei region of South Sudan [19]. Conventionally, cattle rustling are, more or less, a nomadic phenomenon. This observation is predicated on two principal assumptions, namely:

1. It takes a nomad to effectively 'drive' a stolen cow or a cow-herd from the graze.

2. It also takes a nomad to efficiently navigate the 'forest routes', escaping with the stolen cow.

Without any prejudice to possible exceptions, it would take an active involvement, or at least connivance, of a nomad to plan and prosecute a 'good' cattle rustling deal.

In Nigeria the situation have even made the herdsmen to migrate south ward causing more problem along their rout and with suspicion on their host communities attack and destroy farm lands compounding the situation on ground. This has made state governments to legislate and make new laws which may not provide permanent solution. In this paper, we have therefore considered the application of Internet of things system that can track the rustlers and provide security check to the problem.

\section{INTERNET OF THINGS}

The Internet of Things (IoT) is an environment in which objects, animals or people are provided with unique identifiers and the ability to transfer data over a network without requiring human-to-human or human-to-computer interaction. IoT has evolved from the convergence of wireless technologies, microelectromechanical systems (MEMS) and the Internet. The concept may also be referred to as the Internet of Everything.

The next wave in the era of computing will be outside the realm of the traditional desktop. In the Internet of Things (IoT) paradigm, many of the objects that surround us will be on the network in one form or another. The IOT concept was coined by a member of the Radio Frequency Identification (RFID) development community in 1999, and it has recently become more relevant to the practical world largely because of the growth of mobile devices, embedded and ubiquitous communication, cloud computing and data analytics[21]. Imagine a world where billions of objects can sense, communicate and share information, all interconnected over public or private Internet Protocol (IP) networks. These interconnected objects have data regularly collected, analyzed and used to initiate action, providing a wealth of intelligence for planning, management and decision making. This is the world of the Internet of Things (IOT)

Since then, many visionaries have seized on the phrase "Internet of Things" to refer to the general idea of things, especially everyday objects, that are readable, recognizable, locatable, addressable, and/or controllable via the Internet, irrespective of the communication means (whether via RFID, wireless LAN, wide- area networks, or other means). Everyday objects include not only the electronic devices we encounter or the products of higher technological development such as vehicles and equipment but things that we do not ordinarily think of as electronic at all [22].

\subsection{RFID and IoT in Chip Implantation}

RFID (Radio Frequency Identification) has existed since the 1940s, used in a device called the IFF (Identification Friend or Foe) system. British Pilots depended on the IFF systems in their planes to distinguish between their own planes and those belonging to German forces [23]. Now reduced to the size of a grain of rice, RFID tags are used in electronic surveillance in various cases. The chip has been inserted into "smart cards" used to automatically charge the user based on a given condition specified in the RFID tracking sensor. The chip can also be used for security purposes. ID cards carrying chips could identify their carriers and distinguish an employee from a non-employee more effectively than a manual checkpoint. Another modern use of the chip is in tracking livestock and pets. These chips are usually "injected beneath the skin" of an animal and used in tracking the animal. This technology has been around for over seventy years but has just become mainstream in various technology applications especially in IoT due to recent low cost. In recent time microchips of the system as illustrated in the sensors discussed early in this work have low cost chips enough to be used for several cows at a well reduced cost. RFID's wireless capability for identification is a powerful capability [23], which reveals both a physical object's nature and location. In the case of the cattle it can serve the purpose of both checking the health of the cattle as well as the location. In this paper we are more concerned with the location. These technology have been existing but it is high-tech and needed to be made usable by the herdsmen who have little or no technological knowledge of its operation. This creates the need for the development of a usable and easy application layer system which will be easy for the herdsmen to use. In this paper, the design of the system is presented with the detail of how the system operates and how they will be used in real life situations among the cattle herdsmen. The design will also present how the system is expected to interact with other users such as the security personnel that will use the system in tracking rustlers.

\subsection{IoT as a Network of Networks}

The RFID within the IoT cannot be effective without the Network, which is made up of a loose collection of disparate, purpose-built interconnectivity. The system will have multiple networks to control signal function, durability features, and control systems, and so on. The network needed to be in a form that rain and other weather changes will be insulated from interrupting the system since the herdsmen live and work within the area that are susceptible to weather changes and the 
elements. Within the IoT system, these networks, and many others, will be connected with added security, analytics, and management capabilities which will allow the system to become even more powerful in what it can help the herdsmen and the security to achieve in checking rustling activities.

The other networks that the IoT needed to function with needed to be in good condition so that the IoT network within the larger network will be very effective.

\section{IOT ARCHITECTURAL DESIGN}

IoT architecture design consists of different suite of technologies supporting IoT system. It illustrate how various technologies relate to each other and communicate the scalability, modularity and configuration of IOT deployments in different scenarios. The functionality of each layer in the architecture is described in figure 3 .

\begin{tabular}{|c|c|c|}
\hline \multicolumn{3}{|c|}{$\begin{array}{l}\text { IoT Application Layer } \\
\text { (Cattle Tracking App) }\end{array}$} \\
\hline \multicolumn{3}{|c|}{ IoT Management Services } \\
\hline $\begin{array}{l}\text { WAN Gateway } \\
\text { Network }\end{array}$ & & $\begin{array}{l}\text { ateway } \\
\text { vork }\end{array}$ \\
\hline \multicolumn{3}{|c|}{ Gateway } \\
\hline Sensor Connectivity ar & & RFID \\
\hline Sensor Activat & & \\
\hline
\end{tabular}

Fig.3. IOT Architectural Design

The architecture clearly show that the system is a stack of different technologies that are more hardwired on the bottom and software based on the top (application layer). It is the top that the users actually interact with and the top in turn interact with the rest of the components at each stage at the bottom of the system. The layers include: the Sensor Activators and the Sensor Connectivity and Network layer supported by the RFID. The next layer is the Gateway layer which can be Local Area Network (LAN) gateway or a Wide Area Network (WAN) gateway depending on the coverage of the system. The WAN Gateway is always preferred since the area of coverage expected for the tracking system is regional in nature. The upper layers are made of the management service layer and the application layer.

\subsection{Sensors Connectivity And Network Layer}

The lowest layer is made up of smart objects integrated with sensors. The sensors enable the interconnection of the cattles and mobile (digital system) allowing real-time information to be collected and processed even when they are embedded into the cattle skin. Most sensors require connectivity to the sensor aggregators (gateways). This can be in the form of a Local Area Network (LAN) such as Ethernet and Wi-Fi connections or Personal Area Network (PAN) such as ZigBee, Bluetooth and Ultra- Wideband (UWB). For sensors that do not require connectivity to sensor aggregators, their connectivity to backend servers/applications can be provided using Wide Area Network (WAN) such as GSM, GPRS and LTE. This is the connectivity preferred in this design since there is availability of GSM signal in most of the areas in review in Nigeria. Many of the GSM operators have equally rolled out high speed $4 \mathrm{G}$ LTE technology which can be easily used in the implementation of the system. Sensors that use low power and low data rate connectivity typically form networks commonly known as wireless sensor networks (WSNs). WSNs are gaining popularity as they can accommodate far more sensor nodes while retaining adequate battery life and covering large areas. The one proposed in this study are equally WSN brand of sensors.

\subsection{Gateways And Network Layer}

Massive volume of data will be produced by these tiny sensors and this requires a robust and high performance wired or wireless network infrastructure as a transport medium. Current networks, often tied with very different protocols, have been used to support machine-to-machine (M2M) networks and their applications. With demand needed to serve a wider range of IOT services and applications such as high speed transactional services, context-aware applications, etc, multiple networks with various technologies and access protocols are needed to work with each other in a heterogeneous configuration. These networks can be in the form of a private, public or hybrid models and are built to support the communication requirements for latency, bandwidth or security.

A possible deployment could consist of a converged network infrastructure that resolves the fragmentation by integrating networks into a single network platform. Converged network layer abstraction allows multiple organizations to share and use the same network independently for their information to be routed without compromising their privacy, security and performance requirements. Each organization thus utilizes the network as if it is a private network resource to them.

\subsection{Management Service Layer}

The management service renders the processing of information possible through analytics, security controls, process modeling and management of devices. One of the important features of the management service layer is the business and process rule engines. IOT brings connection and interaction of objects and systems together providing information in the form of events or contextual data such as temperature of goods, current location and traffic data. Some of these events require filtering or routing to post-processing systems such as capturing of periodic sensory data, while others require response to the immediate situations such as reacting to emergencies on patient's health conditions. The rule engines support the formulation of decision logics and trigger interactive and automated processes to enable a more responsive IOT system. In the area of analytics, various analytics tools are used to extract relevant information from massive amount of raw data and to be processed at a much faster rate. Analytics such as inmemory analytics allows large volumes of data to be cached in random access memory (RAM) rather than stored in physical disks. In-memory analytics reduces data query time and augments the speed of decision making. Streaming analytics is another form of analytics where analysis of data, considered as data-in-motion, is required to be carried out in real time so that decisions can be made in a matter of seconds. For example, this requirement is typical in the transportation sector where realtime traffic information enables drivers to optimize their routes and travelling times. Analytics can be carried out at other layers within the IOT architecture. For example, analytics may 
be carried out in the smart object layer, i.e., local hub or edge device, so that subsets of the information can be carried through the network for further processing. At this layer, analytics helps to reduce the stress placed on the network layer, reduce power needs of sensors by less frequent communication backend and allow faster responses to data received by the sensors.

Data management is the ability to manage data information flow. With data management in the management service layer, information can be accessed, integrated and controlled. Higher layer applications can be shielded from the need to process unnecessary data and reduce the risk of privacy disclosure of the data source. Data filtering techniques such as data anonymization, data integration and data synchronization, are used to hide the details of the information while providing only essential information that is usable for the relevant applications. With the use of data abstraction, information can be extracted to provide a common business view of data to gain greater agility and reuse across domains.

Lastly, security must be enforced across the whole dimension of the IOT architecture right from the smart object layer all the way to the application layer. Security is of the utmost importance as the integrity of the data must be protected as data travels across the entire system. The integrity of data enables reliable and authentic decisions to be made. Moreover, security of the system prevents system hacking and compromises by unauthorized personnel, thus reducing the possibility of risks.

\subsection{Application Layer}

There are various applications from industry sectors that can leverage on IOT. Applications can be verticalised if they are specific to a particular industry sector, and other applications such as Fleet Management, Asset Tracking, and Surveillance which can cut across multiple industry sectors, but in our case cattle tracking is the area of application.

\section{DESIGN OF THE APPLICATION LAYER}

\subsection{Monitoring Cow!}

In the design of our system we illustrate how cows will be monitored by herdsmen using the application designed and deployed in the mobile devices. Herdsmen first implants sensors in the skin of cattle.

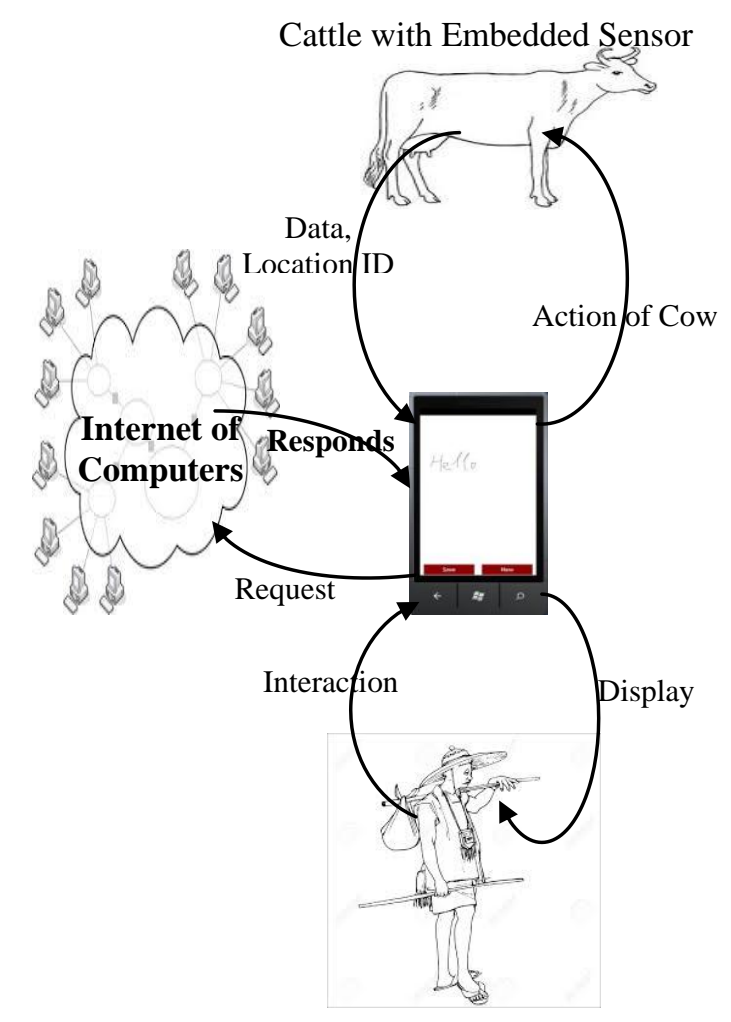

Fig. 4: An IoT Anti-Cattle Rustling System Design

The sensors communicate with the mobile device via the communication system's application layer. The mobile device on the other hand communicates with the cloud via the internet system which allows the herdsmen gadgets to work effectively with the sensors. The herdsmen can then monitor the cows' health and track their movements using the application in the mobile devices which we expect will be highly user-friendly.

\subsection{IOT Applications Design}

The connecting of various non-computer objects with people and computers to the Internet created IoT which offers a huge window of opportunity opened for the creation of applications in the areas of automation, sensing, and machine-to-machine communication. The IoT use applications which need to be designed in such a way that it will be user friendly and usable by the target user (Herdsmen) for the development of the system. In the design of the system certain factors are taking into consideration.

i) The educational level of the users.

ii) Their ability to dial a phone number and successfully initiate a phone communication.

iii) They should know the reason for the system and be willing to accept its usage.

iv) If there is a need to learn how to use the gadget the learning process should be minimal in terms of time, cost and manpower.

v) The users and there environment needed to be carefully evaluated and taking into consideration.

The system can be easily illustrated using a UML object diagram as shown in figure 5 . The application at the application layer in an android or other platforms power the Tracking 
service which in turn assist the alarm on mobile phone to trigger when prompted or when there is an incident of rustling.

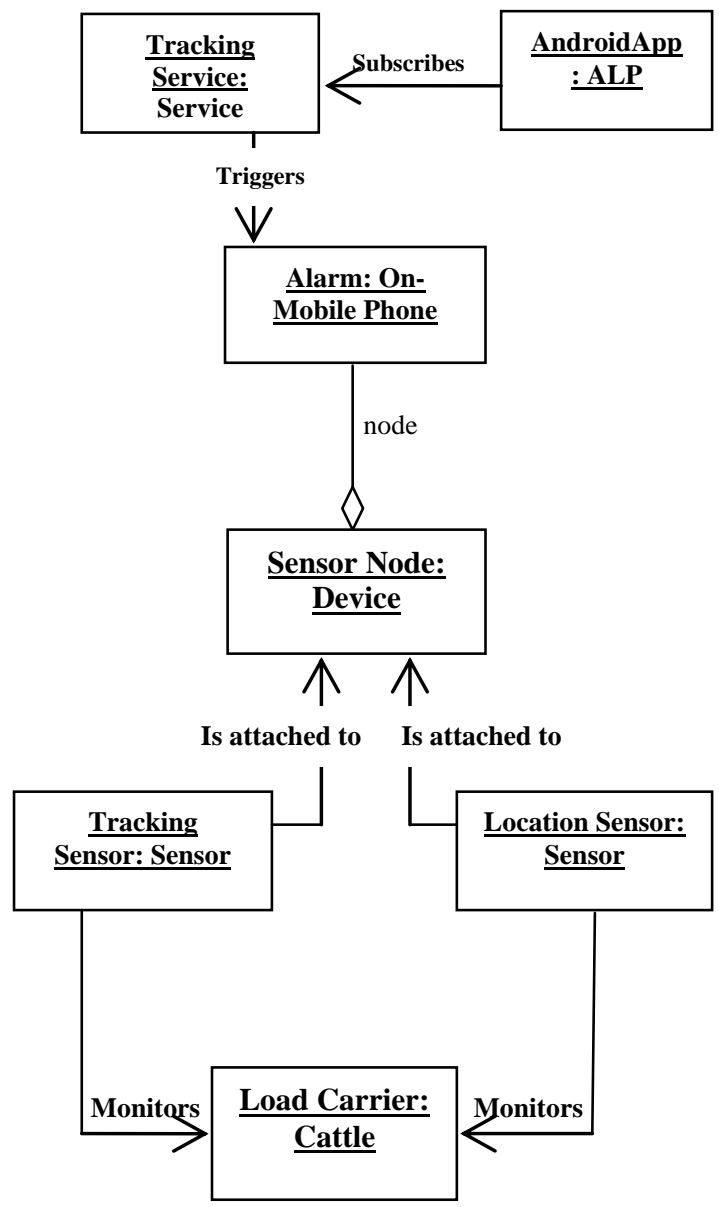

Fig. 5: UML object design of the IoT System

The trigger of the alarm is made possible by the setting of the sensor node which is attached to both the location and tracking part of the sensor used in monitoring the cattle in the field. The way that the objects in the system interact with each other is clearly shown in the UML object design of the IoT system in figure 5 .

In the class design diagram of the IoT System for cattle rustling checking, presented in figure 6 , the classes design for the system development is presented. The users made up of the Herdsmen, GSM providers and the Security Men expected to respond when the system is triggered are clearly shown as they interact with the sensors embedded in the Cattle. The tracking service is also linked to the sensor embedded in the cattle.

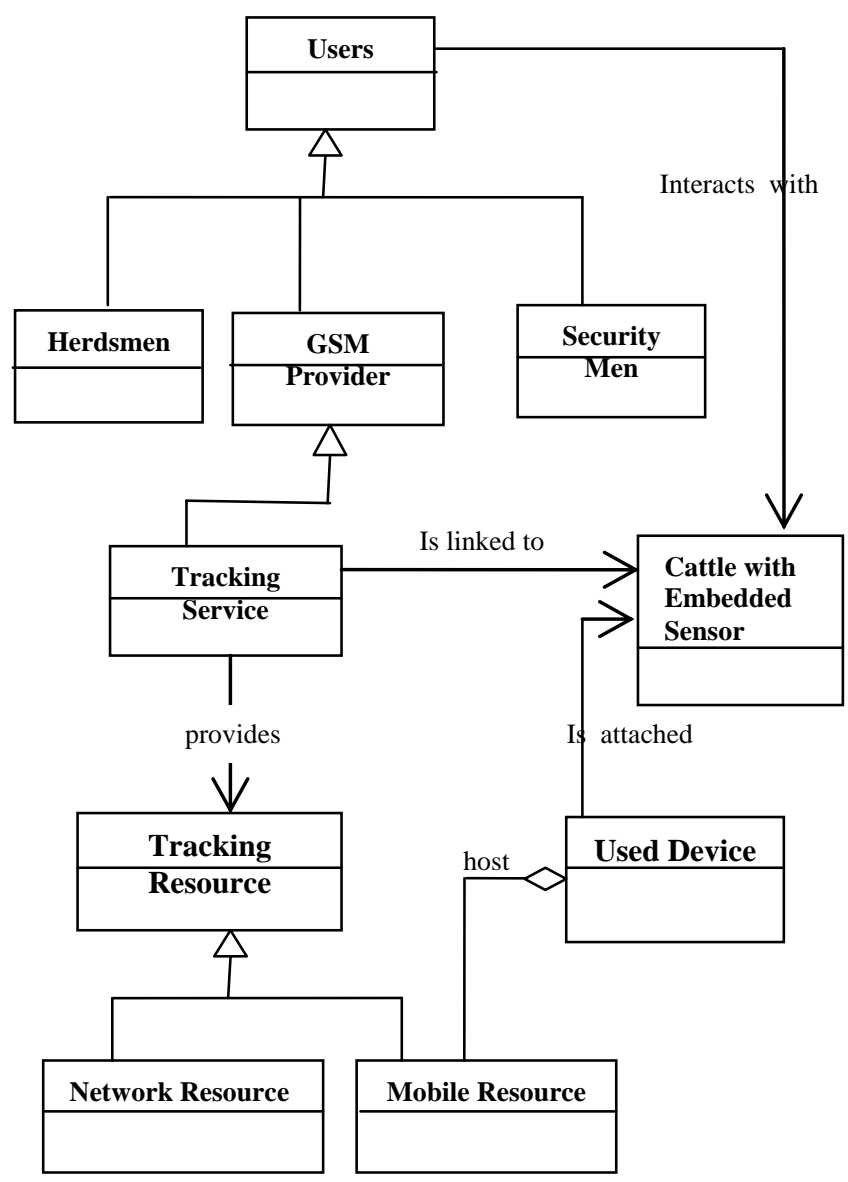

Fig. 6: A Class Design of the IoT System

In the design, it is clear that the tracking service system have the internet part that the embedded sensor needs to be able to effectively communicate with the GSM devices. It is basically derived from the GSM provider and it is dependent on the technology available to the provider at the point of implementation. The Tracking services provides the tracking resources that is extended by the mobile resource class and the network resource class. The mobile resources in turn are an aggregate of the mobile device used in connecting to the attached sensor inside the cattle in the system. The embedded sensor interacts with the users in the system making the system completely interactive. The interaction between the classes provide a simple design that can be used in the implementation of the system. The details of the technology of the GSM provider may vary from $3 \mathrm{G}$ technology to $4 \mathrm{G}$ LTE technology depending on what is available on the network and the cost of the services. These two issues may be a trade-off during implementation the latest technology may be used at a higher cost or the moderate one at a minimum cost. The technology used for the implementation not withstanding the result based on the design will still be effective, since all GSM technologies are relatively working well in the region of our coverage. The deployment must take into consideration that it is not only the loss of the cattle that is our concern but the wellbeing of the herdsmen and the cost implication of the security apparatus that will be used in maintaining peace if the rustling activities results to communal clash and war.

\section{CONCLUSION}

In this paper a design of internet of things system, that when implemented, will assist in checking cattle rustling in Nigeria have been proposed. This research was embarked on considering the increased cases of stealing of grazing cattle in 
Nigeria among the herdsmen. The rustling activity provided an excuse to the herdsmen to start carrying dangerous weapons which they even use in harassing farmers. Many soldiers were deployed to safeguard cattle, creating high costs for the government. This cost can be avoided when the system proposed in this paper is deployed and also safeguard activities of the herdsmen. The design is simple but done with the intent of using the available resources to provide an Internet of Things (IoT) system capable of solving the local problem in the region. In the design, an environment is created which provide the cattle, the herdsmen, the GSM providers and the security personnel with unique identifiers and the ability to transfer data over a network without requiring human-to-human or humanto-computer interaction. The design also allow the cattle tracking service derived from the GSM provider to interface with the sensor embedded in the skin of the cattle. It allow the tracking of resource in the form of network resource and mobile resource which is attached to the mobile device. The System triggers when the cattle are forcefully moved away from its location, once the user set it on. It also performs tracking even after the cattle have been taken away and the system is latter activated. In all the cases the herdsmen or users must interact with the sensor in other to be able to trigger the system. The auto-trigger is not added, due to some design, usage and economic reasons. The main reason being that the battery in the sensor need to be continuously in ON state to be able to achieve auto-trigger which will drain the system battery and make the system sensor to be replaced regularly or to be ineffective when the emergency delays. The user based trigger design in the system has the advantage of preserving the sensor battery until real treat actually occur, then the battery can be used throughout the search and rescue period. The system will ensure that when rustling occurs the application can be used in tracking the criminals. The various state governments were most of the herdsmen operation is carried out can take up the design proposed in this paper and see to its full implementation. In the future the implemented system will not only track cattle but other farm animals and endangered species that are often invaded illegally by various hoodlums who are bent on destroying the environment or the farmers animal.

\section{RECOMMENDATION}

The research is recommended to the developers of IoT systems who can take cue from the design in building their own system. The developers who will need the design in the implementation of their system will also find the work very useful. Researchers who are working in other system may also get inspiration from the system design in developing other systems which may not necessarily be an IoT but system that require some of the object-oriented design pattern used in the development of the system. The work is also recommended to other researcher who would need to leverage on the system to build more advanced system by either improving the sensor or other hardware technology required in the development of the advanced IoT systems. Programmers can also use the design to improve develop varying code for the implementation of the system using different internetworking technology to be able to be emphatic in the process of recommendation of a specific technology for use in field deployment. In that case our implementation will form a bases for well informed comparison of the situation.

\section{ACKNOWLEDGMENT}

We thank Justina Jonah of JavaHouse whose steady discussion of the activities of herdsmen in rural farms in Nigeria lead to this research. We also acknowledgment the contribution of Oyol
Computer Consult, Inc Staff in providing us with their office resources in typing and editing the paper.

\section{REFERENCES}

[1] Steve M. (2003). Toward a Global "Internet of Things". In:SunDeveloperNetwork.2003.http://java.sun.com/develo per/technicalArticles/Ecommerce/rfid/

[2] Sven S., Gregor B., Massimo P., Enrico R., John H., Matthias W. and Albrecht S., (2016) Mobile Interaction with the Internet of Things ,Embedded Interaction Research Group, Media Informatics Group, University of Munich

[3] Dale J. and Cory J.(2016) What does Mobile Broadband System, Techopedia, https://www.techopedia.com/definition/8265/mobilebroadband-system-mbs

[4] Boehm, B.W. (1991) Software Risk Management: Principles and Practices. IEEE Software, 8, 32-41. http://dx.doi.org/10.1109/52.62930

[5] Michael Rohs, Beat Gfeller. Using Camera-Equipped Mobile Phones for Interacting with Real-World Objects. In: Alois Ferscha, Horst Hoertner, Gabriele Kotsis (Eds.): Advances in Pervasive Computing, Austrian Computer Society (OCG), ISBN 3-85403-176-9, pp. 265-271, Vienna, Austria, April 2004

[6]. Rukzio, E., Wetzstein, S., Schmidt, A.: A Framework for Mobile Interactions with the Physical World. Invited paper special session "Simplification of user access to ubiquitous ICT services" at the Wireless Personal Multimedia Communication (WPMC'05) conference, Sept 18-22, 205 - Aalborg, Denmark.

[7]PhysOrg (2011) First Practical Nanogenerator Produces Electricitywith Pinch of the Fingers," PhysOrg.com, March 29, 2011,http://www.physorg.com/news/2011-03nanogenerator-electricity-fingers.html

[8] Alan E. (2016) Mini Tracker Circuit, Mini tracker Project, http://www.talkingelectronics.com/projects/MiniTracker/ TrackingTx.html

[9 ] Teare, Diane (July 1999).Designing Cisco Networks. Indianapolis: Cisco Press.

[10] Okoli, A. C. and Okpaleke, F. N.(2014) Cattle Rustling and Dialectics of Security in Northern Nigeria, International Journal of Liberal Arts and Social Science Vol. 2 No. 3 April, 2014

[11] Blench, R (2004). Natural resources conflict in NorthCentral Nigeria: A Handbook and case studies. Mallam Dendo Ltd.

[12] Alemika, E.E (Ed.) (2013). The impact of organised crime on governance in West Africa. Abuja: FriedrichEbert-Stiftung (Abuja Regional Office, Nigeria).

[13] Okoli, A.C and Agada, A.T. (2014). Kidnapping and national security in Nigeria. Research on Humanities and Social science, 4(6), pp 137-146.

[14] Okoli, A.C and Atelhe, G.A (2014). Nomads against Natives: A political ecology of herder/farmer conflicts in Nasarawa State, Nigeria. American International Journal of Contemporary Research, (2), pp.76-88.

[15] Cheserek G.J, Omondi P, and Odenyo V.A.O (2012) Nature and Causes of Cattle Rustling among some 
Pastoral Communities in Kenya, Journal of Emerging Trends in Economics and Management Sciences (JETEMS) 3 (2): 173-179, Scholarlink Research Institute Journals, 2012.

[16] Greiner. C (2013). Guns, Land, and Votes: Cattle rustling and politics of boundary (re)making in Northern Kenya. African Affairs: Oxford; doi:10.109/afraf/ad+003 (accessed May, 2016).

[17] Gueye, A.B. (2013). Organized crime in the Gambia, Guinea-Bissau and Senegal. In E.E.O. Alemika (Ed.), The impact of organized crime on governance in West Africa. Abuja: Friedrich-Ebert-Stiftung. (Abuja Regional Office, Nigeria).

[18] Alemika, E.E.O (2013). Organized crime and governance in West Africa: Overview in E.E.O Alemika (Ed.), The Impact of organized crime on governance in West Africa. Abuja: Friedrich-Ebert-Stiftung (Abuja Regional Office, Abuja).
[19] Okoli, Al Chukwuma and Okpaleke, Francis N. (2014) Cattle Rustling and Dialectics of Security in Northern Nigeria, International Journal of Liberal Arts and Social Science Vol. 2 No. 3 109-117.

[20] IRIN (2013). Bandit attacks displace northern Nigerian herders.http://www.irinew.org/fr/report/98251/banditattacks-dispalce-northern-Nigeria-herders (accessed May, 2016).

[21] Want, R. (2005) An Introduction to RFID Technology. IEEEPervasive Computing Magazine 5.( 1), 25-33.

[22] Michael R. and Beat G.(2004). Using Camera-Equipped Mobile Phones for Interacting with Real-World Objects. In: Alois Ferscha, Horst Hoertner, Gabriele Kotsis (Eds.) Advances in Pervasive Computing, Austrian Computer Society (OCG), ISBN 3-85403-176-9, pp. 265-271, Vienna, Austria.

[23] Crispo B., Melanie R. R., and Tannenbaum A. S..(2005)The Evolution of RFID Security." IEEEPervasive Computing Magazine 5. (1) 62-69 